\title{
A Particle Swarm Optimization for Reactive Power and Voltage Control in Electric Power Systems
}

\author{
Yoshikazu Fukuyama, Shinichi Takayama, Yosuke Nakanishi \\ Power Engineering Development Lab. \\ Fuji Electric Corporate R \& D, Ltd. \\ No. 1, Fuji-machi, Hino-city, Tokyo,191 Japan \\ Email: fukuyama-yoshikazu@fujielectric.co.jp
}

\author{
Hirotaka Yoshida \\ Technical Research Center \\ The Kansai Electric Power Co., Inc. \\ 3-11-20, Nakoji, Amagasaki, Hyogo, 661 Japan
}

\begin{abstract}
This paper presents a particle swarm optimization (PSO) for reactive power and voltage control (Volt/Var Control: VVC) in electric power systems considering voltage security. VVC can be formulated as a mixed-integer nonlinear optimization problem (MINLP). The proposed method expands the original PSO to handle a MINLP and determines an on-line VVC strategy with continuous and discrete control variables such as automatic voltage regulator (AVR) operating values of generators, tap positions of onload tap changer (OLTC) of transformers, and the number of reactive power compensation equipment. The method considers voltage security using a continuation power flow (CPFLOW) and contingency analysis technique. The feasibility of the proposed method is demonstrated and compared with reactive tabu search (RTS) and the enumeration method on practical electric power system models with promising results.
\end{abstract}

\section{INTRODUCTION}

One of the important operating tasks of power utilities is to keep voltages within an allowable range for high quality customer service. Electric power loads varies from hour to hour and voltages can be varied by the power load change. Power utility operators in control centers control various equipment such as generators, transformers, static condenser (SC), and shunt reactor (ShR), so that they can inject reactive electric powers and control voltages directly in target power systems in order to follow the load change. VVC determines an on-line control strategy for keeping voltages of target power systems considering the load change and reactive power balance in target power systems.

Current practical VVC in control centers is usually realized based on electric power flow sensitivity analysis of the operation point using limited execution time and available data from the actual target power system. Reduction of power generation cost is one of the current interested issues of power utilities. Therefore, optimal control to minimize power transmission loss is required for VVC instead of simple power flow sensitivity analysis. Since many voltage collapse accidents have been occurred over the last three decades (Van Cutsem, 1998), voltage security problem has been dominated and the consideration of the problem has been required in VVC problem (Van Cutsem, 1995, Thukaram, 1996). Two evaluation should be performed to consider voltage security. First one is to calculate the distance between the current operating point and a voltage collapse (saddle node bifurcation) point. The calculation can be realized by a drawing P-V curve using CPFLOW (Chiang, 1995). The authors has been developed a practical CPFLOW program (Yoshida, 1998). Another one is to suppose various faults for the current operating point in the target power system and calculate the distance between the post-fault operating points and voltage collapse points for each fault. The calculation is called voltage contingency analysis (Van Cutsem, 1998). If sufficient distance can be kept for both calculation, the calculated new operating condition can be evaluated as a secure condition. Thus, the advanced VVC requires an optimal control strategy considering power loss minimization and voltage security.

VVC can be formulated as a MINLP with continuous state variables such as AVR operating values and discrete state variables such as OLTC tap positions and the number of reactive power compensation equipment (SC, ShR, etc). The objective function can be varied according to the power system condition. For example, the function can be minimization of power transmission loss of the target power system for the normal operating condition as described above. Conventionally, the methods for VVC problem have been developed using various methods such as fuzzy, expert system, mathematical programming, and sensitivity analysis (Tomsovic, 1992, Cova, 1995, Ramos, 1995, Wu, 1995, Vu, 1996, Le, 1997). However, a practical method for a VVC problem formulated as a MINLP with continuous and discrete state variables has been eagerly awaited.

PSO is one of the evolutionary computation (EC) techniques (Kennedy, 1995). The method is improved and applied to various problems (Shi, 1998, Zhenya, 1998, 
Kennedy, 1998, Angeline, 1998). The original method is able to handle continuous state variables and it can be expanded to handle both continuous and discrete variables easily. Therefore, the method can be applicable to a VVC formulated as a MINLP. Various methods have been developed for a MINLP such as generalized benders decomposition (GBD) (Geoffrion, 1972) and OA/ER (Kocis, 1989). PSO is easy to apply to various problem compared with these methods. Moreover, VVC requires various constraints that are difficult to be handled by mathematical ways. PSO is expected to be suitable for VVC because it can handle such constraints easily.

This paper presents a PSO for a VVC problem formulated as a MINLP considering voltage security. Voltage security is considered using CPFLOW and a contingency analysis technique. The feasibility of the proposed method for VVC is demonstrated and compared with RTS (Battiti, 1994, Fukuyama, 1998) and the enumeration method on practical electric power system models with promising results.

The rest of the papers is organized as follows: In section 2, problem formulation of VVC is described. PSO and CPFLOW are explained in section 3 and 4 briefly. In section 5, formulation of VVC using PSO is explained. In section 6, The proposed method is compared with RTS and enumeration method using electric power systems including practical systems. The paper is concluded with a short summary of the results and their significance.

\section{PROBLEM FORMULATION OF VVC}

\subsection{PROBLEM FORMULATION}

VVC for a normal power system condition can be formulated as follows:

minimize $f_{c}(x, y)=\sum_{i=1}^{n}$ Loss $_{i}$

where, $\mathrm{n}$ : the number of branches,

$\mathrm{x}$ : continuous variables,

$\mathrm{y}$ : discrete variables,

Loss $_{\mathrm{i}}$ : power loss (ploss) at branch $\mathrm{i}$,

subject to

(1) Voltage constraint

Voltage magnitude at each node must lie within their permissible ranges to maintain power quality.

(2) Power flow constraint

Power flow of each branch must lie within their permissible ranges.

(3) Voltage security

The Determined VVC strategy should keep voltage security of the target power system.

Ploss of the target power system is calculated for a certain VVC strategy using load flow calculation with both continuous variables (AVR operating values) and discrete variables (OLTC tap positions and the number of reactive power compensation equipment). Load flow calculation calculates voltages and currents of the target power systems using various electric power load characteristics. Load flow equations are formulated as a set of simultaneous nonlinear equations and usually solved by the iterative methods such as Newton-Raphson method (Arrilaga, 1990). Voltage and power flow constraints can be checked at the load flow calculation and penalty values are added if the constraints are violated. P-V curve for the determined VVC strategy and various contingencies can be generated and checked whether the VVC candidate can keep sufficient voltage security margins.

\subsection{STATE VARIABLES}

The following control equipment is considered in the VVC problem.

(1) AVR operating values (continuous variable)

(2) OLTC tap position (discrete variable)

(3) The number of reactive power compensation equipment (discrete variable)

Each generator has AVR function and it can keep the terminal voltage of each generator. Terminal voltages are set to a certain value to regulate reactive power output from the generators. Transformer has OLTC function, automatic tap position changer, to regulate the secondary side voltage. Reactive power compensation equipment such as SC and ShR is installed to inject and absorb reactive powers.

The state variables are treated in load flow calculation as follows: AVR operating values are treated as voltage specification values. OLTC tap positions are treated as tap ratio to each tap position. The number of reactive power compensation equipment is treated as corresponding susceptance values.

\section{OVERVIEW OF PARTICLE SWARM OPTIMIZATION}

PSO has been developed through simulation of simplified social models. The features of the method are as follows:

(1) The method is based on researches on swarms such as fish schooling and bird flocking.

(2) It is based on a simple concept. Therefore, the computation time is short and it requires few memories.

(3) It was originally developed for nonlinear optimization problems with continuous variables. However, it is easily expanded to treat problems with discrete variables. Therefore, it is applicable to a MINLP with both continuous and discrete variables such as VVC.

The above (3) is suitable for the VVC problem because practically efficient methods have not been developed for VVC with both continuous and discrete variables. The above features allow PSO to handle the VVC problem and require few computation time.

The research results for bird flocking indicate that birds find food by flocking (not by each individual). The observation leads the assumption that every information is shared inside 
flocking. According to the observation of behavior of human groups, behavior of each individual (agent) is also based on behavior patterns authorized by the groups such as customs and other behavior patterns according to the experiences by each individual. The assumption is a basic concept of PSO. PSO is basically developed through simulation of bird flocking in two-dimension space. The position of each agent is represented by XY-axis position and the velocity is expressed by vx (the velocity of $\mathrm{X}$-axis) and vy (the velocity of Y-axis). Modification of the agent position is realized by the position and velocity information.

Searching procedures by PSO based on the above concept can be described as follows: A flock of agents optimize a certain objective function. Each agent knows its best value so far (pbest) and its XY position. Moreover, each agent knows the best value in the group (gbest) among pbests. Each agent tries to modify its position using the current velocity and the distance from pbest and gbest. The modification can be represented by the concept of velocity. Velocity of each agent can be modified by the following equation:

$$
\begin{aligned}
& v_{i}^{k+1}=v_{i}^{k}+\text { rand } \times\left(\text { pbest }_{i}-s_{i}^{k}\right)+\text { rand } \times\left(\text { gbest }-s_{i}^{k}\right) \\
& \text { where, } v_{i}{ }^{k} \quad \text { : velocity of agent } \mathrm{i} \text { at iteration } \mathrm{k} \text {, } \\
& \text { rand : random number between } 0 \text { and } 1 \text {, } \\
& \mathrm{s}_{\mathrm{i}}{ }^{\mathrm{k}} \quad \text { : current position of agent } \mathrm{i} \text { at iteration } \mathrm{k} \text {, } \\
& \text { pbest }_{\mathrm{i}} \text { : pbest of agent } \mathrm{i} \text {, } \\
& \text { gbest : gbest of the group. }
\end{aligned}
$$

Using the above equation, a certain velocity which gradually gets close to pbest and gbest can be calculated. The current position (searching point in the solution space) can be modified by the following equation.

$$
s_{i}^{k+1}=s_{i}+v_{i}^{k+1}
$$

Fig. 1 shows the above concept of modification of searching points. PSO utilizes several searching points like genetic algorithm (GA) and the searching points gradually get close to the optimum using their pbests and gbest. The features of the searching procedure can be summarized as follows:

(1) Initial positions of pbest and gbest are different. However, using the different direction of pbest and gbest, all agents gradually get close to the optimum.

(2) The modified value of the agent position is continuous and the method can be applied to the continuous problem. However, the method can be applied to the discrete problem using grids for $\mathrm{XY}$ position and its velocity easily.

(3) There are no inconsistency in searching procedures even if continuous and discrete state variables are utilized with continuous axes and discrete grids for XY positions and velocities. Namely, the method can be applied to a MINLP with continuous and discrete state variables naturally and easily.
(4) The above concept is explained using only XY axis (2dimension space). However, the method can be easily applied to n-dimension problem.

The original PSO has been applied to a learning problem of neural networks and Schaffer f6, the famous benchmark function for GA, and efficiency of the method has been confirmed (Kennedy, 1995).

\section{OVERVIEW OF CONTINUATION POWER FLOW}

P-V curve represents the relation between load increase and voltage drop. It can be calculated by increasing total electric loads in the target power system gradually and plotting the dropped voltage. CPFLOW utilizes system loads as parameters and calculates a $\mathrm{P}-\mathrm{V}$ curve by modification of the parameters using the continuation method. It is one of the methods in applied mathematics and calculates transition of equilibrium points (eg. P-V curve) by modification of parameters. In order to avoid the illcondition around the nose (saddle node bifurcation) point, an arclength along the $\mathrm{P}-\mathrm{V}$ curve is introduced as an additional state variable and the electric power flow equation is expanded. The continuation method is applied to the expanded equation and the P-V curve can be generated rapidly without ill-condition around the nose point. CPFLOW can generate a P-V curve automatically and can be applied to large-scale power systems easily (Chiang, 1995, Yoshida, 1998).

The proposed method generates a P-V curve using the CPFLOW technique and calculates a MW margin, distance between the current operating point and the saddle node bifurcation point, for the determined control strategy. The proposed method also utilizes the fast voltage contingency analysis method using CPFLOW (Chiang, 1997). Then, the method checks whether the MW margin is enough or not, compared with the predetermined value. Using the procedure, the method checks whether the target power system can keep voltage security by the control or not.

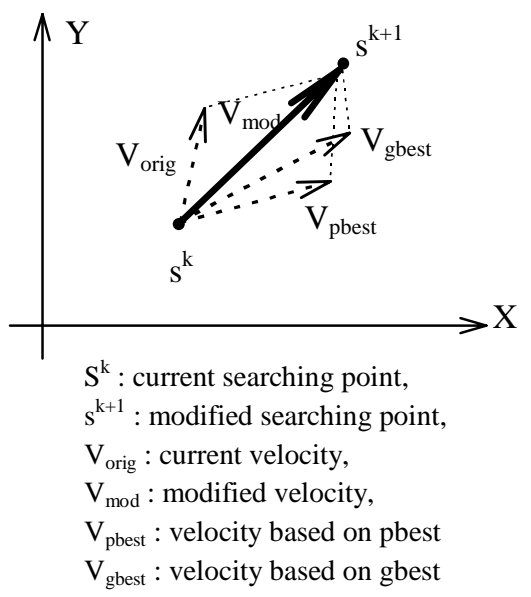

Fig.1 Concept of modification of a searching point. 


\section{FORMULATION OF VVC USING PSO \\ 5.1 TREATMENT OF STATE VARIABLES}

Each variable is treated in PSO as follows: Initial AVR operating values are generated randomly between upper and lower bounds of the specification values. The value is also modified in the search procedure between the bounds. OLTC tap position is initially generated randomly between the minimum and maximum tap positions. The value is modified in the search procedure among existing tap positions. Then, the corresponding impedance of the transformer is calculated for load flow calculation. The number of reactive power compensation equipment is also generated from 0 to the number of existing equipment at the substation initially. The value is also modified in the search procedure between 0 and the number of existing equipment.

\subsection{VVC ALGORITHM USING PSO}

The proposed VVC algorithm can be expressed as follows:

Step 1. Initial Searching points (agents) and velocities are generated using the above-mentioned state variables randomly.

Step 2. Ploss to the searching points for each agent is calculated using the load flow calculation. If the constraints are violated, penalty is added to the loss (evaluation value of agent).

Step 3. Pbest is set to each initial searching point. The initial best evaluated value (loss with penalty) among pbests is set to gbest.

Step 4. Velocities are calculated using eqn. (2).

Step 5. New searching points are calculated using eqn. (3).

Step 6. Ploss to the new searching points and the evaluation values are calculated.

Step 7. If the evaluation value of each agent is better than the previous pbest, the value is set to pbest. If the best pbest is better than gbest, the value is set to gbest. All of gbests are stored as candidates for the final control strategy.

Step 8. If the iteration number reaches to the maximum number, then go to Step 9. Otherwise, go to Step 4.

Step 9. P-V curves for the control candidates and various contingencies are generated using the best gbest among the stored gbests (candidates). If the MW margin is larger than the predetermined value, the control is determined as the final solution. Otherwise, select next gbest and repeat the procedure.

\section{NUMERICAL EXAMPLES}

\subsection{IEEE 14 BUS SYSTEM}

\subsubsection{Simulation conditions}

Fig. 2 shows a modified IEEE standard 14 bus system. The followings are control variables.
(1) Continuous AVR operating values of node 2,3,6, and 8: Upper and lower bounds are 0.9 and 1.1 [pu].

(2) Discrete tap positions of transformers between node 4-7, 4-9, and 5-6: These transformers are assumed to have 20 tap positions.

(3) Discrete number of installed SC in node 9 and 14: Each node is assumed to have three 0.06 [pu] SC.

The proposed method generates an optimal control for the original operating condition with Ploss as 0.1349 [pu].

Generation of the VVC candidates (Step 1 - 7 in the proposed VVC algorithm) by the proposed PSO based method, RTS, and the enumeration method is compared in the simulation. The number of agents for PSO is 10 . The initial tabu length is 10 and increase/decrease rate for tabu length is 0.2 for RTS in the simulation. The results are compared with 300 searching iterations. RTS and the enumeration method utilizes digitized AVR operating values and the interval is 0.01 [pu]. The interval corresponds to $5[\mathrm{kV}]$ in $500[\mathrm{kV}]$ system. The formulation as the combinatorial optimization problem has about $10^{9}$ combinations in the problem.

\subsubsection{Simulation results}

PSO is one of the stochastic optimization method and it should be evaluated statistically. Fig. 3 shows the statistical evaluation result of the proposed method by 100 trials. Table 1 shows the results by the proposed method (best), RTS and the enumeration method. The best result by PSO is better than both RTS and the enumeration method. One of the tap position is different between PSO formulated as a MINLP , and RTS and the enumeration method formulated as combinatorial optimization problem. PSO can generate the loss value under the optimal value by RTS with $10 \%$ possibility. The calculation time of PSO is about twice faster than that of RTS. The result indicates that VVC should be formulated as a MINLP and the suitability of the

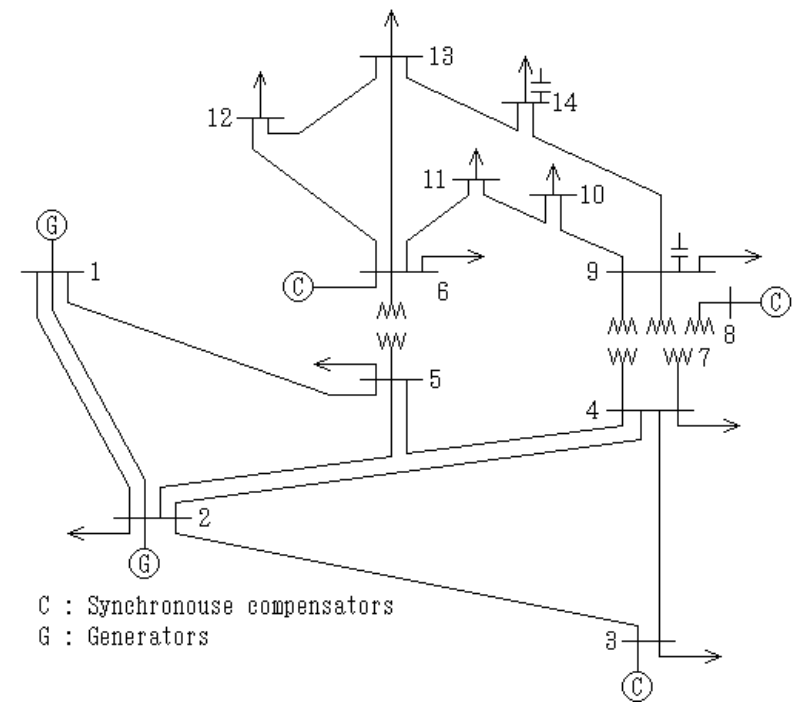

Fig. 2 A modified IEEE 14 bus system. 
PSO for VVC problem.

The proposed method generates a P-V curve for the optimal control strategy using the CPFLOW technique and performs the voltage contingency analysis. It is verified that the strategy can keep voltage security.

\subsection{PRACTICAL MODEL SYSTEM WITH 112 BUSES}

\subsubsection{Simulation conditions}

The proposed method is applied to the practical model system with 112 buses. The model system has 11 generators for AVR control, 47 OLTCs with 9 to 27 tap positions, and 33 SCs for VVC.

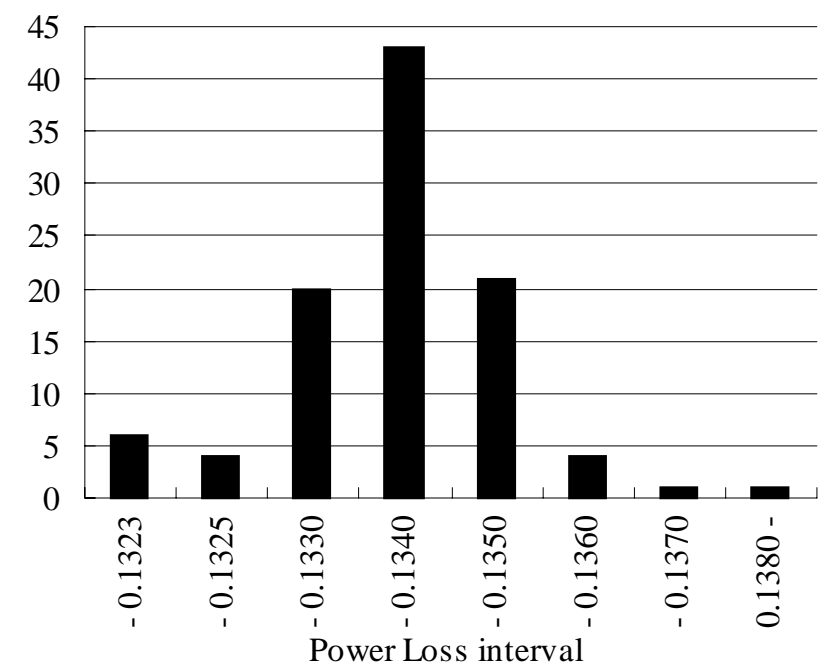

Fig. 3 Statistical results by PSO (100 trials).

Table 1 The optimal control for IEEE 14 bus system.

\begin{tabular}{|l|c|c|c|}
\hline $\begin{array}{l}\text { Mont. } \\
\text { Variables }\end{array}$ & PSO & RTS & $\begin{array}{l}\text { enumeration } \\
\text { method }\end{array}$ \\
\hline AVR 2 & 1.0463 & 1.05 & 1.05 \\
\hline AVR 3 & 1.0165 & 1.02 & 1.02 \\
\hline AVR 6 & 1.1000 & 1.10 & 1.10 \\
\hline AVR 8 & 1.1000 & 1.10 & 1.10 \\
\hline Tap 4-7 & 0.94 & 0.95 & 0.95 \\
\hline Tap 4-9 & 0.93 & 0.93 & 0.93 \\
\hline Tap 5-6 & 0.97 & 0.97 & 0.97 \\
\hline SC 9 & 0.18 & 0.18 & 0.18 \\
\hline SC 14 & 0.06 & 0.06 & 0.06 \\
\hline Loss & 0.132276 & 0.132366 & 0.132366 \\
\hline Cal. Time [s] & 30 & 54 & - \\
\hline
\end{tabular}

AVR 2 : AVR operating values [pu] at node 2

Tap 4 - 7 : Tap ratio between node 4 and 7

SC 9 : Susceptance [pu] at node 9

Loss : power loss [pu]

Cal. Time : For 300 iterations using PC (Pentium 400MHz, FreeBSD ver. 3.0, GCC)

\subsubsection{Simulation results}

Fig. 4 shows typical convergence characteristics (Ploss transition of gbest by PSO and the best result by RTS). It is clear from the figure that the solution by PSO is converged to a high quality solution at the early iterations (about 20 iterations). On the contrary, RTS reaches the best result gradually. The fast convergence characteristic of PSO is always observed in 10 trials. Table 2 shows the best results by PSO and RTS. PSO always generates better results compared with RTS in 10 trials. Calculation time for 20 iterations by PSO is less than 3 [min] and this is short enough to use the proposed method for practical VVC. The determined VVC strategy is evaluated as secure in voltage using a CPFLOW technique. The above results reveal that the suitability of the proposed method for practical VVC.

\section{CONCLUSIONS}

This paper presents a particle swarm optimization (PSO) for reactive power and voltage control (VVC) considering voltage security. The proposed method formulates VVC problem as a mixed integer nonlinear problem (MINLP) and determines control strategy with continuous and discrete control variables such as AVR operating values, OLTC tap positions, and the number of reactive power compensation equipment. The method also considers voltage security using a continuation power flow (CPFLOW) technique. The feasibility of the proposed method for VVC is demonstrated on practical power systems with promising results. The results can be summarized as follows:

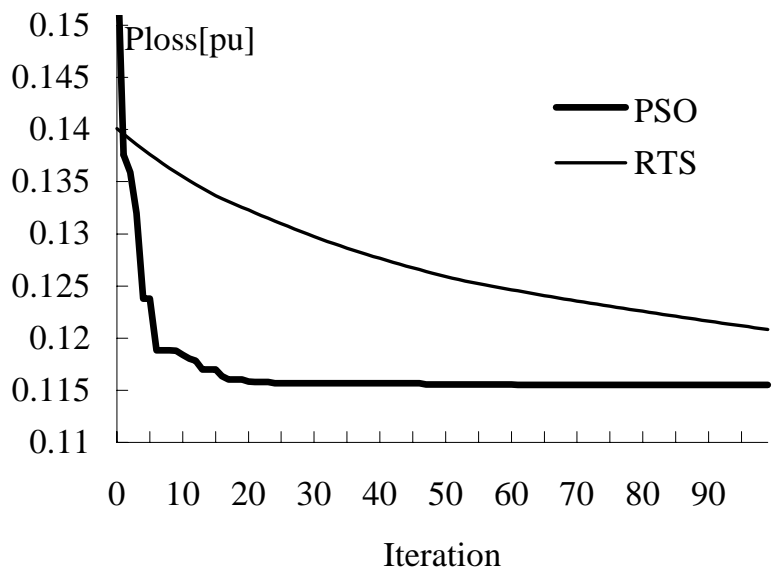

Fig. 4 Convergence characteristics by PSO and RTS for practical 112 bus system.

Table 2 Calculation results by PSO and RTS for practical 112 bus system.

\begin{tabular}{|l|l|l|}
\hline & PSO & RTS \\
\hline Loss [pu] & 0.1155 & 0.1208 \\
\hline Cal. Time [s] & 804 & 6465 \\
\hline
\end{tabular}

* Cal. Time is for 100 iterations using PC (Pentium 400MHz, FreeBSD ver. 3.0, GCC). 
(1) Discrete variables of the optimal result formulated as a MINLP and those formulated as a combinatorial optimization problem is different as shown in the results. VVC is usually formulated as a combinatorial optimization problem. However, the problem should be formulated as a MINLP.

(2) The proposed PSO based VVC can generate reasonable results for the application to practical power systems and it is suitable for practical VVC.

(3) Consideration of voltage security is one of the important practical functions of VVC. The results reveal that the possibility of treatment of the security by the proposed PSO-based method in VVC.

Considering more practical features such as avoidance of control concentration to a specific equipment, tracking to load change, and look-ahead control using load forecast, the proposed method will be improved considering the above features for the future works.

\section{REFERENCES}

T. Van Cutsem, C. Vournas (1998). Voltage Stability of Electric Power Systems. Kluwer Academic Publishers.

T. Van Cutsem (1995). An Approach to Corrective Control of Voltage Instability Using Simulation and Sensitivity. IEEE Transaction on Power Systems. 10(2):616-622.

B. D. Thukaram, K. Parthasarathy (1996). Optimal Reactive Power Dispatch Algorithm for Voltage Stability Improvement. International Journal of Electrical Power \& Energy Systems. 18(7):461-468.

H. D. Chiang, et al. (1995). CPFLOW: A Practical Tool for Tracing Power System Steady-State Stationary Behavior Due to Load and Generation Variations. IEEE Transaction on Power Systems. 10(2):623-634.

H. Yoshida, Y. Fukuyama, et al. (1998). Practical Continuation Power Flow for Large-Scale Power System Analysis. Proceedings of IEE of Japan Annual Convention Record, No. 1313 (in Japanese).

K. Tomsovic (1992). A Fuzzy Linear Programming Approach to the Reactive Power / Voltage Control Problem. IEEE Transaction on Power Systems. 7(1):287-293.

B. Cova, et al. (1995). Contingency Constrained Optimal Reactive Power Flow Procedures for Voltage Control in Planning and Operation. IEEE Transaction on Power Systems. 10(2):602-608.

J. L. M. Ramos, et al. (1995). A Hybrid Tool to Assist the Operator in Reactive Power / Voltage Control and Operation. IEEE Transaction on Power Systems. 10(2):760768.

Q. H. Wu, et al. (1995). Power System Optimal Reactive
Power Dispatch Using Evolutionary Programming. IEEE Transaction on Power Systems. 10(3):1243-1249.

H. Vu, et al. (1996). An Improved Voltage Control on Large-Scale Power System. IEEE Transaction on Power Systems. 11(3):1295-1303.

T. L. Le et al. (1997). Network Equivalents and Expert System Application for Voltage and VAR Control in Large - Scale Power Systems. IEEE Transaction on Power Systems. 12(4):1440-1445.

J. Kennedy, R. Eberhart (1995). Particle Swarm Optimization. Proceedings of IEEE International Conference on Neural Networks. IV:1942-1948.

Y. Shi, R. Eberhart (1998). A Modified Particle Swarm Optimizer. Proceedings of IEEE International Conference on Evolutionary Computation. 69-73.

H. Zhenya, et al. (1998). Extracting Rules from Fuzzy Neural Network by Particle Swarm Optimization. Proceedings of IEEE International Conference on Evolutionary Computation. 74-77.

J. Kennedy, W. Spears (1998). Matching Algorithm to Problems: An Experimental Test of the Particle Swarm Optimization and Some Genetic Algorithms on the Multimodal Problem Generator. Proceedings of IEEE International Conference on Evolutionary Computation. 78-83.

P. Angeline (1998). Using Selection to Improve Particle Swarm Optimization. Proceedings of IEEE International Conference on Evolutionary Computation. , 84-89.

A. M. Geoffrion (1972). Generalized Benders Decomposition. Journal of Operation Theory and Applications. 10(4):237-260.

G. R. Kocis, I. E. Grossmann (1989). Computational Experience with DICOP solving MINLP Problems in Process Systems Engineering. Computer Chemical Engineering. 13(3):307-315.

R. Battiti (1994). The Reactive Tabu Search. ORSA Journal of Computing. 6(2):126-140.

Y. Fukuyama, et al. (1998). A Reactive Tabu Search for Service Restoration in Electric Power Distribution Systems. Proceedings of IEEE International Conference on Evolutionary Computation. 763-768.

J. Arrilaga, C. P. Arnold (1990). Computer Analysis of Power Systems. John Wiley \& Sons.

H. D. Chiang, et al. (1997). Look-ahead Voltage and Load Margin Contingency Selection Functions for Large-scale Power Systems. IEEE Transaction on Power Systems. 12(1):173-180. 